\title{
IL-23 is essential for T cell-mediated colitis and promotes inflammation via IL-17 and IL-6
}

David Yen, ${ }^{1}$ Jeanne Cheung, ${ }^{1}$ Heleen Scheerens, ${ }^{1}$ Frédérique Poulet, ${ }^{2}$ Terrill McClanahan, ${ }^{3}$ Brent Mckenzie, ${ }^{1}$ Melanie A. Kleinschek, ${ }^{1}$ Alex Owyang, ${ }^{1}$ Jeanine Mattson, ${ }^{3}$ Wendy Blumenschein, ${ }^{3}$ Erin Murphy, ${ }^{3}$ Manjiri Sathe,${ }^{3}$ Daniel J. Cua, ${ }^{1}$ Robert A. Kastelein, ${ }^{1}$ and Donna Rennick ${ }^{1}$

1Department of Discovery Research, Schering-Plough Biopharma, Palo Alto, California, USA. ${ }^{2}$ Schering-Plough Research Institute, Lafayette, New Jersey, USA. 3Department of Experimental Pathology and Pharmacology, Schering-Plough Biopharma, Palo Alto, California, USA.

\begin{abstract}
Uncontrolled mucosal immunity in the gastrointestinal tract of humans results in chronic inflammatory bowel disease (IBD), such as Crohn disease and ulcerative colitis. In early clinical trials as well as in animal models, IL-12 has been implicated as a major mediator of these diseases based on the ability of anti-p40 mAb treatment to reverse intestinal inflammation. The cytokine IL-23 shares the same p40 subunit with IL-12, and the anti-p40 mAbs used in human and mouse IBD studies neutralized the activities of both IL-12 and IL-23. IL-10-deficient mice spontaneously develop enterocolitis. To determine how IL-23 contributes to intestinal inflammation, we studied the disease susceptibility in the absence of either IL-23 or IL-12 in this model, as well as the ability of recombinant IL-23 to exacerbate IBD induced by T cell transfer. Our study shows that in these models, IL-23 is essential for manifestation of chronic intestinal inflammation, whereas IL-12 is not. A critical target of IL-23 is a unique subset of tissue-homing memory $T$ cells, which are specifically activated by IL-23 to produce the proinflammatory mediators IL-17 and IL-6. This pathway may be responsible for chronic intestinal inflammation as well as other chronic autoimmune inflammatory diseases.
\end{abstract}

\section{Introduction}

When mucosal immunity is not countered by antiinflammatory mediators (e.g., IL-10 or TGF- $\beta$ ), excessive proinflammatory responses result in chronic inflammatory bowel disease (IBD) (1). To investigate the mechanism(s) responsible for IBD, we have studied 2 murine models: (a) IL-10-KO mice, which spontaneously develop enterocolitis (2) resembling Crohn disease and (b) lymphocyte-deficient Rag-KO mice, which develop colitis after reconstitution with $\mathrm{CD}^{+} \mathrm{T}$ cells from $I L-10-\mathrm{KO}$ mice $(3,4)$. The intestinal disease that occurs in these models is initiated by the excessive generation of IFN- $\gamma$-producing T cells (Th1) driven by IL-12 produced by antigenpresenting cells. Thus, early treatment with anti-IFN- $\gamma \mathrm{mAb}(2,4)$ or anti-IL-12(p40) mAb prevented disease (4). In contrast, treatment with anti-IL-12(p40) mAb, but not anti-IFN- $\gamma \mathrm{mAb}$, reversed ongoing disease in both of our models (4) and in a chemically induced colitis model (5). Based on these outcomes, it was concluded that IL-12 might play a role in colitis independent of its ability to generate IFN- $\gamma$ producing T cells. However, recent studies have indicated that IL-23, which, like IL-12, is produced by antigen-presenting cells, and is also inhibited by anti-p $40 \mathrm{mAb}$, regulates autoimmune-inflammatory processes in several mouse disease models (6-8). Support for a significant role of p40-containing cytokines (IL-12 and/or IL-23) in the pathogenesis of human disease comes from 2 early clinical trials (on Crohn disease and psoriasis) using p40-specific human mAbs $(9,10)$. In both trials, administration of anti-p40 resulted in improved clinical disease, but it was not clear whether this was due to neutralization of IL-12 or IL-23. To define the role of IL-12 versus IL-23 in chronic intestinal inflammation, we have conducted studies using $I L$-10-deficient mice to measure the individual contributions of these related cytokines.

Nonstandard abbreviations used: IBD, inflammatory bowel disease. Conflict of interest: The authors have declared that no conflict of interest exists. Citation for this article: J. Clin. Invest. 116:1310-1316 (2006). doi:10.1172/JCI21404.

\section{Results}

$I L-12 p 35 \times I L-10-K O$ but not IL-23p19 $\times$ IL-10-KO mice develop spontaneous IBD. To determine the relative contributions of IL-12 and IL-23 in IBD, we backcrossed IL-10-KO mice with mice lacking only $I L-12\left(p 35^{-/-}\right)$or only IL-23 $\left(p 19^{-/-}\right)$. As observed previously, $I L-10-\mathrm{KO}$ mice developed colitis by 3 months of age (2). At 12 months, half of the IL-10-KO colony had wasted and died, and $100 \%$ of the survivors exhibited severe colitis (Figure 1, A and lower panel of C). Similarly to the $I L-10$-deficient animals, $I L-12 p 35 \times$ IL-10-KO mice developed signs of colitis, including diarrhea and rectal prolapse as early as 7 weeks of age. Histological evaluation of colons from these animals at 3 months of age revealed marked intestinal inflammation, ruling out that IL-12 is required for the development of spontaneous IBD in $I L-10$-deficient animals (Figure 1B). However, when $I L-10-\mathrm{KO}$ mice were backcrossed with p19-KO mice, the $I L-10 \times p 19$-double-KO mice were still disease free at 12 months of age (Figure 1, A and upper panel of C). This result suggested that IL-23, but not IL-12, is required for the manifestation of chronic intestinal inflammation. CD4 ${ }^{+} \mathrm{T}$ cells from $I L-10$ $\times$ p19-KO mice still produced large amounts of IFN- $\gamma$, indicating that $I L-10 \times p 19-\mathrm{KO}$ mice are not impaired in their ability to generate a Th1 cell response (Figure $2 \mathrm{~A}$ ). The level of IFN- $\gamma$ produced by $\mathrm{CD}^{+} \mathrm{T}$ cells from $\mathrm{IL}-10 \times p 19-\mathrm{KO}$ mice consistently appeared increased over that of the $I L-10-\mathrm{KO} \mathrm{CD}^{+} \mathrm{T}$ cells, suggesting an enhanced Th1 response in $I L-10 \times p 19-\mathrm{KO}$ mice. In contrast, $\mathrm{CD} 4^{+}$ $\mathrm{T}$ cells from $I L-10 \times p 35-\mathrm{KO}$ mice showed reduced levels of IFN- $\gamma$ (Figure 2B) Similar results were recently seen in the pathogenesis of 2 other chronic inflammatory autoimmune diseases, EAE and collagen-induced arthritis (CIA). In both diseases, Th1 responses developed normally in the absence of IL-23, but disease manifestation required the presence of IL-23.

Recombinant IL-23 accelerates T cell transfer colitis. To assess how IL-23 may be enhancing IBD, we performed studies in a T cell transfer model of colitis. The recipients of passively transferred $\mathrm{T}$ cells 
A

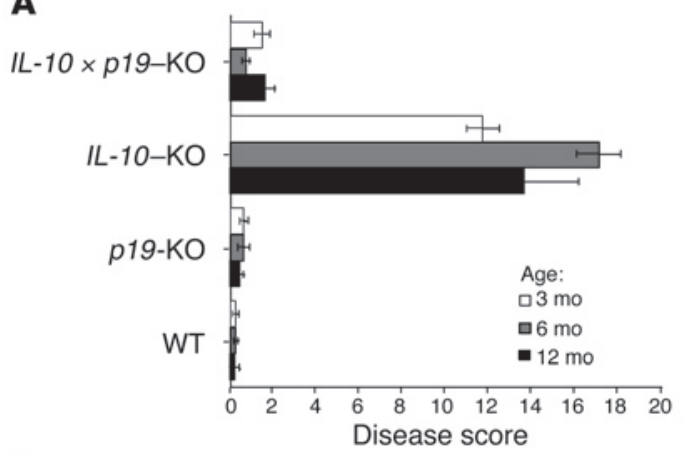

B

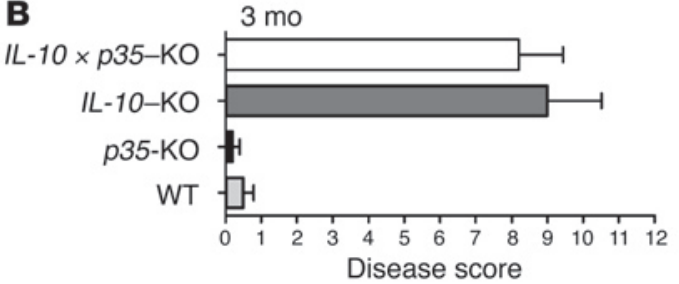

C
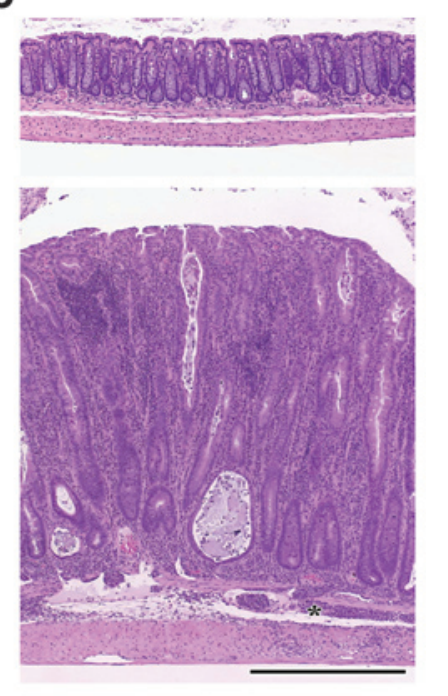

Figure 1

IL-23, but not IL-12, is essential for spontaneous colitis induced by IL-10 deficiency. (A) Histologic changes were evaluated at 3 time points in the colons of mice that were $I L-10$ deficient and/or IL-23 ( $p 19)$ deficient. (B) Histologic changes were evaluated at 3 months in the colons of mice that were $I L-10$ deficient and/or IL-12 (p35) deficient. The disease scores for each group $(n=5-8)$ were obtained as previously described (41). (C) Photomicrographs of the descending colons from 12month-old IL-10 × p19-KO mice (upper panel) and $I L-10-K O$ mice (lower panel). The lower panel shows marked mucosal thickening and epithelial hyperplasia. *Inflammation extended into the submucosa and tunica muscularis. Scale bar: $50 \mu \mathrm{m}$. were Rag-KO mice, which are devoid of mature $\mathrm{T}$ and $\mathrm{B}$ cells. They normally develop colitis $10-12$ weeks after reconstitution with either naive $\mathrm{T}$ cells $\left(\mathrm{CD} 4^{+} \mathrm{CD} 45 \mathrm{RB}^{\text {high }}\right)$ or with memory $\mathrm{T}$ cells $\left(\mathrm{CD} 4{ }^{+} \mathrm{CD} 45 \mathrm{RB}^{\text {low }}\right)$ from diseased $I L-10-\mathrm{KO}$ mice $(3,4)$. However, recipients that were treated daily with IL-23 developed colitis after only 4 weeks (Figure 3A). The accelerated onset of colitis occurred regardless of whether IL-23-treated Rag-KO mice were reconstituted with naive or memory $\mathrm{CD}^{+} \mathrm{T}$ cells. IL-23 treatment also led to splenomegaly and a blood neutrophilia $\left(4,800 \pm 800 / \mathrm{mm}^{3}\right.$ of blood), whereas saline-treated controls still had normal spleens and baseline neutrophil counts $\left(1,500 \pm 200 / \mathrm{mm}^{3}\right.$ of blood). The mesenteric LNs of IL-23-treated recipients contained greatly expanded numbers of $\mathrm{CD}^{+}{ }^{+} \mathrm{T}$ cells and $\mathrm{CD} 11 \mathrm{~b}^{-} \mathrm{CD} / \mathrm{c}^{+} \mathrm{F} 4 / 80^{-} \mathrm{DCs}$ (Figure 3B). Continuous infusions with IL-23 did not result in colitis in unreconstituted Rag-KO mice.

IL-23 promotes memory-activated T cell production of IL-6 and IL-17. In order to better define the actions of IL-23, gene expression studies were performed to investigate the mechanism by which IL-23 induces colitis using the $T$ cell transfer model. For the gene expression analysis, gene-specific primers were used in TaqMan quantitative RT-PCR to detect the modulation of chemokines, cytokines, and cell-associated activation molecules. Gene expression of colon samples from recipients treated for 4 weeks with IL-23 (colitis) was compared with that of saline-treated controls (no colitis) and naive controls (no cell transfer). Many genes were upregulated following IL-23 treatment, which signified the influx of activated inflammatory macrophages (i.e., $I L-1 \beta, T N F-\alpha, N O S-2)$ and granulocytes (i.e., myeloperoxidase, 12-lipoxygenase) (data not shown). In addition, increased expression of monocyte chemoattractant protein-1 and monokine induced by IFN- $\gamma$ (Figure 4A) as well as MMP-7 and MMP-12 (data not shown) may contribute to digestion of basement membranes and matrix proteins and the migration of cells into the mucosa. We also found that IL-23 treatment increased gene expression specific to T cells (CD3 $\varepsilon$ chain; Figure 4A), confirming the rapid infiltration of colons by donor $\mathrm{CD}^{+} \mathrm{T}$ cells. Early T cell engraftment was accompanied by increased gene expression for IFN- $\gamma$, which had already been linked to colitis in this model $(2-4,11)$. In addition, IL-23 treatment also resulted in increased IL-17 gene expression (Figure 4A).
Although IFN- $\gamma$ can be made by accessory cells and T cells, IL-17 is predominantly made by $\mathrm{T}$ cells (12). Previous studies have shown that IL-17 is produced preferentially by human and murine $\mathrm{T}$ cells with a memory/activated phenotype $(13,14)$. To further examine the role of IL-23 in activation of memory T cells, sorted $\mathrm{CD}^{+}{ }^{+} \mathrm{CD} 45 \mathrm{RB}^{\text {low }}$ memory $\mathrm{T}$ cells from $\mathrm{IL}-10-\mathrm{KO}$ mice were assessed for cytokine gene expression following stimulation in the presence or absence of IL-23. As shown in Figure 4B, the mRNA levels of TNF and IFN- $\gamma$ were unchanged by IL-23. In contrast, IL-17 gene expression was elevated by IL-23. In parallel experiments (data not shown), it was found that IL-23 had no effect on gene expression by naive $T$ cells, consistent with their failure to express the IL-23 receptor (15). In addition to elevation of IL-17 gene expression, IL-23 also strongly induced gene expression of IL-6 (Figure 4B).

Memory T cells from WT and mutant mice were then assayed for cytokine secretion using ELISA. In the presence of plate-bound anti-CD3 mAb, IL-23 specifically stimulated increased IL-17 production (Figure 5A). The highest level of IL-17 was induced by IL-23 in cells from $I L-10-\mathrm{KO}$ mice. IL-17 was also produced by cells from $I L-10 \times p 19-\mathrm{KO}$ mice but was very low in cells from WT and $p 19-\mathrm{KO}$
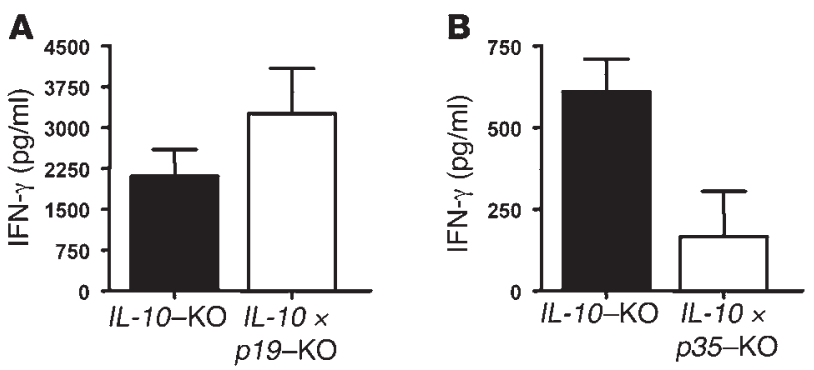

\section{Figure 2}

Normal production of IFN- $\gamma$ in the absence of IL-23, but not IL-12. CD4+ splenocytes from $I L-10$-deficient mice and mice doubly deficient for $I L-10$ and IL-23p19 (A) or IL-12p35 (B) were stimulated with anti-CD3 and anti-CD28 for 3 days. Supernatants were assessed for IFN- $\gamma$ as described in Methods. Bars represent 3 mice per group; error bars indicate SD. Data shown are representative of 2 experiments. 
A

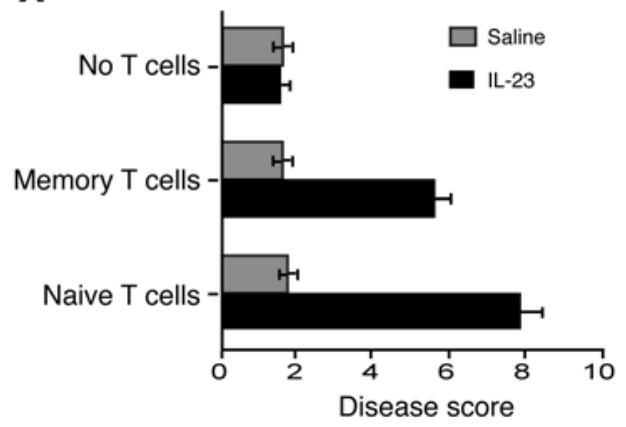

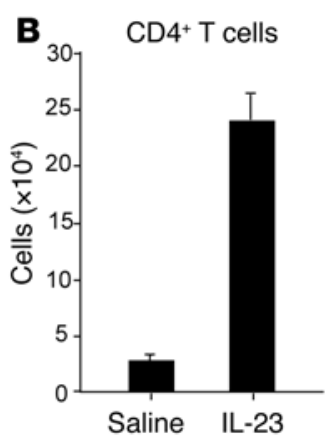

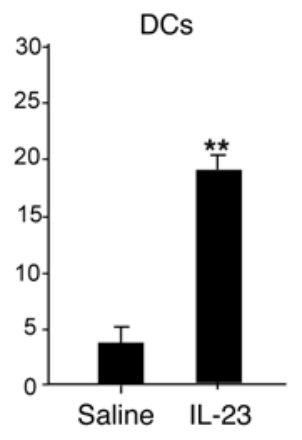

Figure 3

IL-23 treatment accelerates the onset of colitis in T cell-reconstituted immunodeficient recipients. (A) Disease scores of Rag-KO mice reconstituted with naive or memory CD4+ $\mathrm{T}$ cells from $\mathrm{L}-10$ $\mathrm{KO}$ donors followed by daily infusions with IL-23 (1 $\mu \mathrm{g} /$ dose) or saline for 4 weeks $(n=8)$. (B) T cell and accessory cell numbers in mesenteric lymph nodes of reconstituted Rag-KO mice treated with IL-23 or saline for 4 weeks. mice. Basal levels of IL-4 and IFN- $\gamma$ were not modulated by either IL-12 or IL-23 costimulation. When we assayed for IL-23dependent proliferation in the presence of anti-IL-2 $\mathrm{mAb}$, we found that memory cells from $I L-10-\mathrm{KO}$ were the best responders (Figure 5B). In contrast, memory $\mathrm{T}$ cells from all 4 strains proliferated equally in cultures supplemented with IL-2 (data not shown). Interestingly, there appeared to be a direct correlation between the ability of cells to produce IL-17 in response to IL-23 and the degree of IL-23-stimulated growth. To understand the correlation between IL-23-induced T cell development and IL-17 production, memory $\mathrm{T}$ cells were prepared for intracellular cytokine staining. Cells from $I L-10-\mathrm{KO}$ mice and $I L-10$ $\times$ p19-KO mice contained distinct populations that could produce IL-4 or IFN- $\gamma$ (Figure 5C). Strikingly, only diseased $I L-10-\mathrm{KO}$ mice had a large number of IL-17-positive cells. The vast majority of the IL-17-positive cells were negative for IL-4 and IFN- $\gamma$ staining, showing that they constitute a subset distinct from classical Th1 and Th 2 memory cells. The small number of IL-17-staining $\mathrm{T}$ cells present in $I L-10 \times p 19-\mathrm{KO}$ mice (Figure 5C) suggests that the generation of this particular $\mathrm{T}$ cell subset can occur in the absence of endogenous IL-23 production. Data presented in Figure 5, A and B, indicate, however, that optimal expansion and IL-17 production require IL-23 stimulation.

Blockade of IL-6 and IL-17 ameliorates $I B D$. Our results suggest that IL-23 promotes production of IL- 6 and IL-17 by memory activated $\mathrm{T}$ cells (Figures 4B and 5) from $I L-10-\mathrm{KO}$ mice with IBD. Many reports have shown that IL-6 plays a crucial role

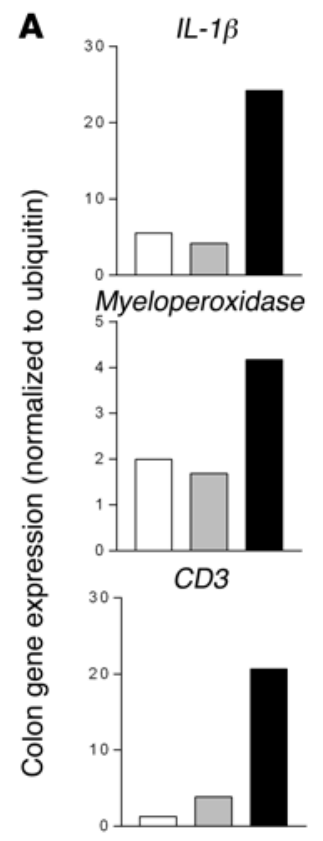

B

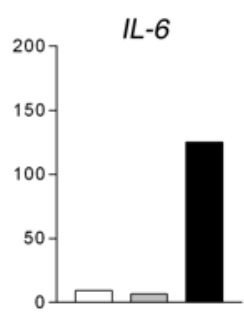

\section{Figure 4}
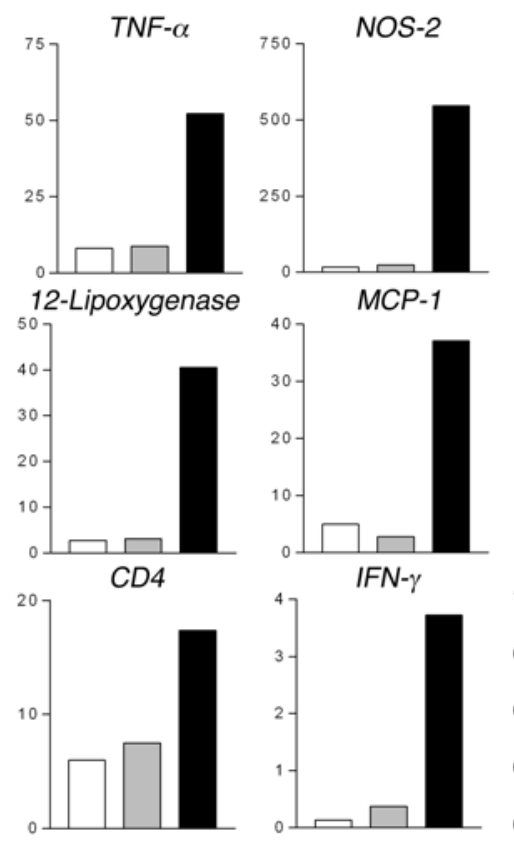

$\mathrm{CD}^{+}$memory activated $\mathrm{T}$ cell
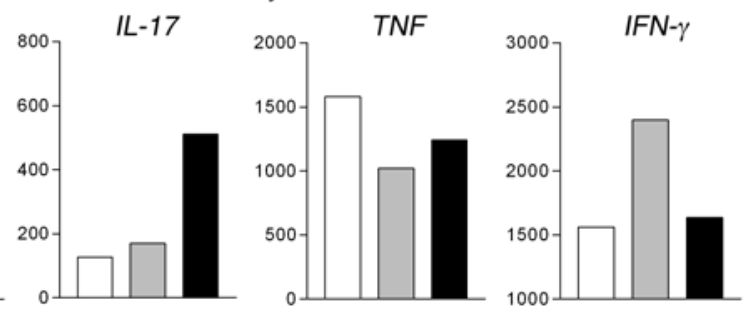

Gene expression induced by IL-23 treatment of Rag-KO mice reconstituted with memory CD4+ $\mathrm{T}$ cells from IL-10-KO mice. (A) Increased mRNA expression in colons of Rag-KO mice reconstituted with memory CD4+ T cells from IL-10-KO mice and treated with IL-23 for 4 weeks. mRNA from the colons of 4 mice were pooled, and gene expression levels were determined by real-time quantitative PCR. Data are presented as values normalized to ubiquitin. (B) Gene expression by memory CD4 ${ }^{+} \mathrm{T}$ cells from IL-10-KO mice following in vitro stimulation with anti-CD3 mAb plus IL-2, IL-12, or IL-23 relative to stimulation with anti-CD3 mAb alone. 
A

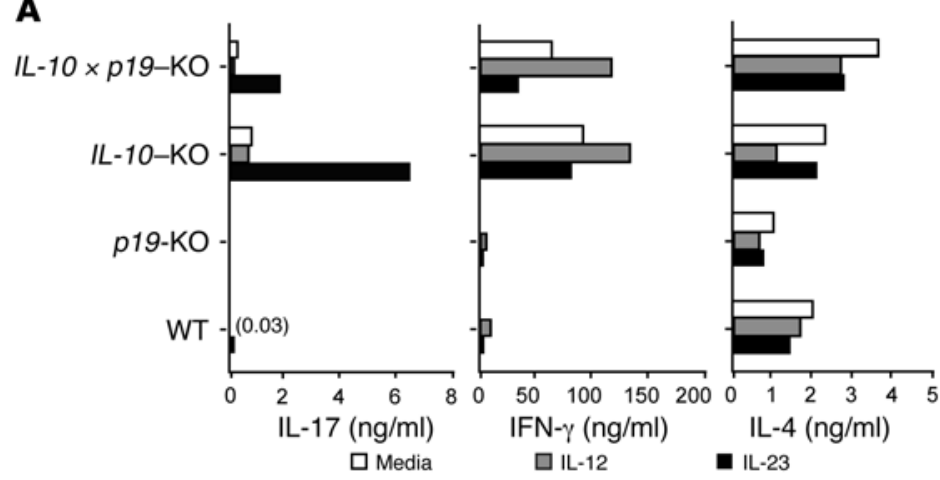

C

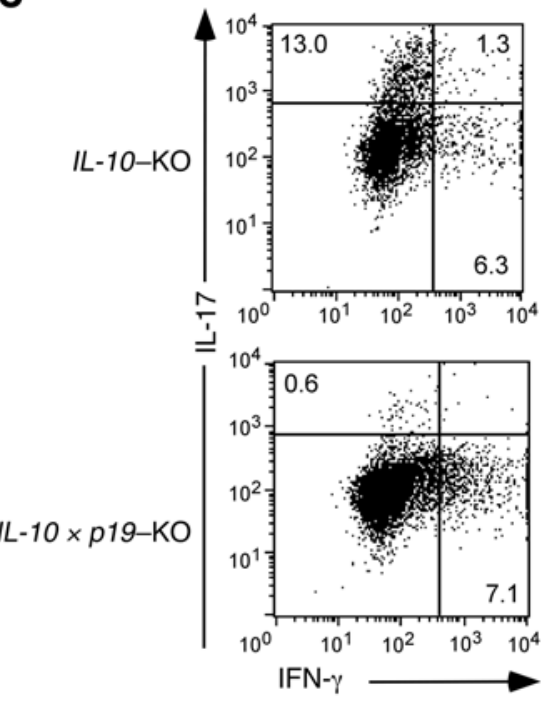

B

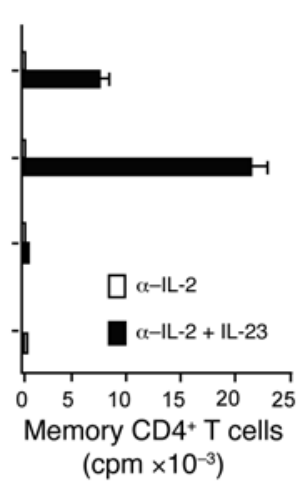

\section{Figure 5}

IL-23, but not IL-12, specifically stimulates a subset of memory $\mathrm{CD}^{+} \mathrm{T}$ cells that produce IL-17. FACS-purified CD4+CD45RBlow splenic T cells $(2 \times 105 / \mathrm{ml} ;>95 \%$ purity) were isolated and cultured on CD3-coated plates. (A) Cytokine production by memory $\mathrm{CD} 4^{+} \mathrm{T}$ cells. Supernatants from cells stimulated with IL-23 $(10 \mathrm{ng} / \mathrm{ml})$ or IL-12 (1 ng/ml) were analyzed by ELISA. (B) IL-23-dependent proliferation of memory CD4 ${ }^{+} \mathrm{T}$ cells was assessed by $\left[{ }^{3} \mathrm{H}\right]$-thymidine incorporation after 4 days of culture on anti-CD3 mAb-coated plates in the presence of anti-IL-2 mAb. (C) FACS analysis of memory CD4+ T cells from $I L-10-K O$ and IL-10 x p19-KO mice after cells were stained for intracellular IL-4, $\mathrm{IL}-17$, and IFN- $\gamma$ proteins.

Heightened IL-6 (19) and IL-17 levels (20) could be detected in both serum and intestinal tissue from patients with Crohn disease. Blocking the IL- 6 signaling pathway prevented the development of $\mathrm{T}$ cell-mediated murine colitis $(16,17)$. Furthermore, several potent proinflammatory factors such as IL-1, TNF, as well as IL-6 are downstream mediators of IL-17 (21). In order to address whether IL-23 induction of IL- 6 and IL-17 production plays a role in the pathogenesis of bowel inflammation, we cotreated T cell-restored recipient mice with IL-23 as well as Abs that neutralize IL-6, IL-17, or both cytokines. Isotype control Ab-treated mice developed enterocolitis (path score $=10$ ) 6 weeks after treatment, while the single Ab-treated groups (anti-IL-6: 6.5 and anti-IL-17: 6) developed an attenuated intestinal inflammation (Figure 6). More importantly, anti-IL-6 and anti-IL-17 combination therapy significantly ameliorated the severity of intestinal inflammation induced by IL-23. Taken together, these results suggest that IL-23 promotes development and expansion of a pathogenic IL-6/IL-17producing memory-activated $\mathrm{T}$ cell population that can trigger the inflammatory cascade leading to intestinal inflammation.

\section{Discussion}

Our studies with IL-23-deficient mice show that IL-23 is essential for the manifestation of intestinal inflammation. This is consistent with the recently described dominant role for IL-23 in both CNS and joint autoimmune inflammation $(6,7)$. Together these findings point to IL-23, but not IL-12, as the necessary mediator of organ-specific autoimmune diseases. Although IL-23 and IL-12 share structural homologies, they have very distinct biological activities. The first striking difference between IL-23 and IL-12 is that IL-23 specifically stimulates memory $\mathrm{CD}^{+} \mathrm{T}$ cells, whereas IL-12 is a potent stimulant for naive $\mathrm{CD}^{+} \mathrm{T}$ cells $(22,23)$. The selective activation of memory T cells by IL-23 is especially relevant to tissue inflammation. Memory $\mathrm{T}$ cells are well known for their tissue-homing properties. In the case of our IBD models, memory $\mathrm{CD}^{+} \mathrm{T}$ cells constitute $60-80 \%$ of the $\mathrm{T}$ cells found in the intestines of mice with colitis (3). Furthermore, reconstitution of immunodeficient SCID or Rag-KO mice with naive CD4+ $T$ cells rapidly leads to the selective expansion and intestinal engraftment of donor $T$ cells expressing a memory phenotype (24). Lamina propria DCs, particularly in the distal end of the small intestine and driven by the intestinal flora, were recently described as constitutively expressing IL-23, suggesting a predisposition of this part of the small intestine to initiate chronic inflammatory responses through IL-23 (25). Interestingly, the constitutive expression of IL-23 in this part of the small intestine was accompanied by a peak in IL-17 expression.

A second important difference is that IL-23, unlike IL-12, induces the production of IL-17 by a unique subset of memory T cells. Therefore, IL-23 regulates a highly potent $\mathrm{T}$ cell-derived cytokine that has broad actions on the immune system. IL-17 is known to stimulate fibroblasts, endothelial cells, macrophages, and epithelial cells to secrete multiple proinflammatory mediators (i.e., 


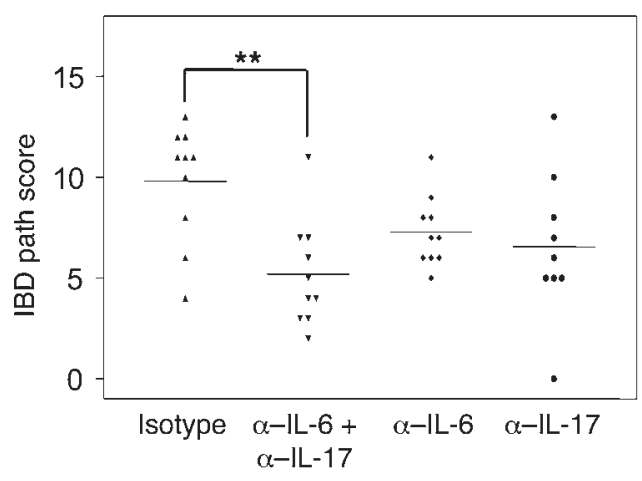

\section{Figure 6}

Blocking IL-6 and IL-17 significantly reduced the intestinal inflammation, by $50 \%$. Recipient mice were dosed i.p. with isotype, anti-IL-6, anti-IL-17, or anti-IL-6 plus anti-IL-17 Abs (2 mg/mouse) a day prior to $\mathrm{T}$ cell reconstitution. Rag-KO mice were reconstituted with sorted splenic CD $4^{+}$CD $45 R B^{\text {hi }}$ (naive) $T$ cells $\left(5 \times 10^{5}\right.$ cells $/$ mouse) from diseased $I L-10-K O$ mice and treated daily with $1 \mu \mathrm{g} / \mathrm{mouse} \mathrm{IL}-23$ protein. Subsequent rounds of Ab were administered weekly for 6 weeks. The graph shows the path scores from 2 independent but identical experiments. The disease scores for each group were obtained as previously described (41). Horizontal bars represent the median value for each group. ${ }^{* *} P<0.05$, compared with isotype Ab (unpaired Student's $t$ test). Histologic examination was performed and scored using formalin-fixed tissue sections stained with $\mathrm{H} \& \mathrm{E}$, as previously described (40).

IL-1, IL-6, TNF, NOS-2, metalloproteases, and chemokines) (14). Importantly, in vivo studies have indicated that the local production of IL-17 may cause a site-specific influx and activation of inflammatory cells. In mice, IL-17 administered into the peritoneal cavity elicited peritonitis (26), and intratracheal administration elicited lung inflammation $(27,28)$. IL-17 has been identified in the synovial fluids of patients with rheumatoid arthritis $(29,30)$ and Lyme disease (31). T cells isolated from the inflamed joints of patients with Lyme disease contained a subset of IL-17-producing $\mathrm{T}$ cells that were distinct from those producing IL- 4 or IFN- $\gamma$. This pattern is similar to what we observed with memory $\mathrm{T}$ cells isolated from mice with colitis. It is also similar to the observations by Murphy et al. in a model of collagen-induced arthritis using IL-23deficient mice (7). Resistance to the development of joint and bone pathology in these mice correlated with an absence of IL-17-producing $\mathrm{CD} 4^{+} \mathrm{T}$ cells despite normal induction of collagen-specific, IFN- $\gamma$-producing Th 1 cells. In separate studies, it was shown that either neutralizing Th1 responses by anti-p $40 \mathrm{mAb}(32-34)$ or blocking IL-17 (35) led to diminished chronic joint inflammation in mice. The connection between these 2 successful treatments was not understood, since IL-17 is not associated with "classical" Th1 cytokine responses (36). Given the data presented here, it is not surprising that both treatments were protective. A similar unique $\mathrm{CD} 4 \mathrm{~T}$ cell activation state characterized by the production of IL-17 in response to IL-23 has recently been observed by Aggarwal et al. (37). Our results show that $\mathrm{CD} 4^{+} \mathrm{T}$ cells from $\mathrm{IL}-10 \times p 19-\mathrm{KO}$ mice still produce high levels of IFN- $\gamma$. If anything, the level of IFN- $\gamma$ produced by cells from these mice appears to be somewhat higher, suggesting an increased Th1 response in these animals in the absence of IL-23, although other Th1 cytokines were largely unchanged. In contrast, $\mathrm{CD} 4^{+} \mathrm{T}$ cells from $\mathrm{IL}-10 \times \mathrm{p} 35-\mathrm{KO}$ mice showed reduced production of IFN- $\gamma$. Since the production of IFN- $\gamma$ is highly dependent on the presence of IL-12, this result was not unexpected. However, despite the reduced level of IFN- $\gamma$, these animals develop colitis very early, while the IL-10 × p19-KO mice remain resistant to colitis development.

Since our studies were conducted in $I L$-10-deficient mice and employed cells in the transfer colitis model obtained from $I L-10$ deficient mice, one could question whether similar results would be obtained in mice with normal IL-10 levels. The absence of IL-10related regulatory effects could account for the increased disease proclivity in these mice. In this context, it is interesting to note that there was increased IL-17 production in IL-10-deficient mice as compared with WT mice (Figure 5A). It is possible that in the presence of normal IL-10 levels, IL-23-mediated inflammation alone would not be sufficient to support mucosal inflammation and would require additional inflammatory signals such as those mediated by IL-12. Several recent EAE and CIA studies, representing commonly used models of chronic inflammation and autoimmunity, have shown that mice deficient in IL-23, but with otherwise normal expression of IL-10, are protected from disease (6-8), making it less likely that our results are only relevant in the absence of IL-10.

Very recently, Langrish et al. (8) have identified a pathogenic IL-23-dependent $\mathrm{T}$ cell population that is essential for the establishment of organ-specific inflammation associated with autoimmunity. This $\mathrm{Th}_{\mathrm{IL}-17}$ population, characterized by the production of IL-17, IL-6, and TNF, can be distinguished from IL-12-driven $\mathrm{T}$ cells with respect to the expression pattern of proinflammatory cytokines and other factors. Furthermore, transfer studies showed that these IL-23-dependent $\mathrm{Th}_{\mathrm{IL}-17} \mathrm{CD} 4^{+} \mathrm{T}$ cells, but not IL-12dependent Th $1 \mathrm{CD}^{+} \mathrm{T}$ cells, are essential for the establishment of inflammation associated with CNS autoimmunity. The data presented here are strikingly similar. $\mathrm{CD} 4^{+} \mathrm{CD} 45 \mathrm{RB}$ low memory $\mathrm{T}$ cells isolated from $I L-10-\mathrm{KO}$ mice produce IL- 6 and IL-17 following stimulation in the presence of IL-23. These cells also produce TNF, but this cytokine is induced in response to both IL-23 and IL-12, suggesting that TNF may play a role in both IL-12- and IL-23linked immune responses. A similar result was obtained after in vitro stimulation of $\mathrm{Th}_{\mathrm{IL}-17}$ and $\mathrm{Th} 1 \mathrm{CD} 4^{+} \mathrm{T}$ cells (8). In addition to IL-17, the production of IL- 6 by these $\mathrm{CD}^{+} \mathrm{CD} 45 \mathrm{RB} \mathrm{B}^{\text {low }}$ memory $\mathrm{T}$ cells is also uniquely dependent on the presence of IL-23. Langrish et al. (8) speculate that IL-6 produced by the pathogenic $\mathrm{Th}_{\mathrm{IL}-17} \mathrm{CD} 4^{+} \mathrm{T}$ cells may in fact inhibit the action of regulatory $\mathrm{T}$ cell function, allowing effector $\mathrm{T}$ cell activation (38). Although the data presented here do not address that question, it is evident that neutralization of IL-6 has a beneficial effect on the IL-23-induced intestinal inflammation. The significant amelioration of disease observed with combined anti-IL- 6 and anti-IL-17 treatment demonstrates that the action of IL- 6 and IL-17, both produced by this pathogenic $T$ cell population, is not redundant and that these 2 inflammatory mediators are to a large extent responsible for the IL-23-driven intestinal inflammation. It is interesting to note that a small number of IL-17-staining T cells remain in IL-10 × p19-KO mice. Similarly, Aggarwal et al. also noted that not all IL-17 production is abrogated in the absence of IL-23 (37).

Although we find that memory $\mathrm{CD}^{+} \mathrm{T}$ cells are a critical target of IL-23 in the development of chronic intestinal inflammation, it is likely not the only cell population that responds to IL-23. In CNS autoimmune inflammation, IL-23 was found to act more broadly as an end-stage effector cytokine through direct actions on macrophages in addition to its effect on memory $\mathrm{CD} 4^{+} \mathrm{T}$ cells $(6,7)$. Other studies have identified DCs as a potential target cell 
population for IL-23 (39). The relative contributions of these cell subsets with respect to IL-23 and disease pathogenesis have not yet been determined.

Our data provide evidence that the activation of tissue-homing memory $\mathrm{T}$ cells by IL-23 is responsible for chronic intestinal inflammatory disease. We show that this cytokine induces a distinct $T$ cell activation state that is characterized by the production of several proinflammatory cytokines, including IL-17 and IL-6, but not IFN- $\gamma$. A similar IL-23-responsive, IL-17/IL-6/TNF-producing activated memory $\mathrm{T}$ cell subset is essential in the pathogenesis of other chronic autoimmune diseases, highlighting the importance of this novel pathway in chronic autoimmune inflammatory disease. This is even more relevant, since recent clinical trials demonstrate improved clinical disease after treatment with anti-p40 Abs $(9,10)$. Since these anti-p40 Abs neutralize both IL-12 and IL-23, it is not clear whether the therapeutic effects are due to neutralization of IL-12 or IL-23. Our data identify IL-23 as the relevant target in colitis.

\section{Methods}

Experimental mice. IL-10-KO mice and littermate $I L-10 \times p 19-\mathrm{KO}, p 19-\mathrm{KO}$ (6), $I L-10 \times p 35-\mathrm{KO}$, and WT mice on the C57BL/6 × 129/SvEv background were generated and maintained at DNAX. C57BL/ 6 mice, Rag-KO mice, and $I L-12(p 40)-\mathrm{KO}$ mice on the BALB/c mouse background as well as $I L-12(p 40)$ - and $I L-12(p 35)-\mathrm{KO}$ mice on the C57BL/6 background were purchased from the Jackson Laboratory. Rag-KO 129/SvEv, IL-10-KO 129/SvEv, and IL-10-KO BALB/c mice were bred at DNAX. All animal procedures were approved by the Schering-Plough Biopharma Institutional Animal Care and Use Committee committee, in accordance with the Association for Assessment and Accreditation of Laboratory Animal Care International.

Histopathology. Histologic examination of colons from IBD experiments was performed by a veterinary pathologist using formalin-fixed tissue sections stained with H\&E, as previously described (40).

$T$ cell reconstitution of Rag-KO mice. Splenic T cells from $I L-10-\mathrm{KO}$ donors were enriched by red cell lysis and magnetic bead depletion using lineage-specific mAb supernatants B220 (B cells), 8C5 (neutrophils), Mac-1 (macrophages), Ter 119 (erythrocytes), and anti-CD8 (BD Biosciences - Pharmingen). Stained cells were removed in a magnetic field using goat anti-rat IgG $(\mathrm{Fc})$ - and anti-rat IgG $(\mathrm{H}+\mathrm{L})$-coated magnetic beads (Perseptive Diagnostics). The remaining cells were stained with CD4-PE and CD45RB-FITC (BD Biosciences - Pharmingen) for sorting on a FACSVantage $\mathrm{SE}(\mathrm{BD}) .5 \times 10^{5} \mathrm{CD}^{+} \mathrm{CD} 45 \mathrm{RB}^{\text {high }}$ (naive) $\mathrm{T}$ cells or $\mathrm{CD}^{+} \mathrm{CD}^{4} 5 \mathrm{RB}^{\text {low }}$ (memory) T cells were injected i.p. into Rag-KO recipients. Recipients were injected i.p. daily with $1 \mu \mathrm{g}$ purified mouse IL-23 (22).

Cytokine and proliferation assays. FACS-purified $\mathrm{CD} 4{ }^{+} \mathrm{CD} 45 \mathrm{RB}{ }^{\text {low }} \mathrm{T}$ cells from the spleen $\left(2 \times 10^{5} / \mathrm{ml} ;>95 \%\right.$ purity) were cultured on CD3-coated plates in medium supplemented with $50 \mathrm{ng} / \mathrm{ml} \mathrm{IL-12} \mathrm{(R \& D} \mathrm{Systems)} \mathrm{or}$ $50 \mathrm{ng} / \mathrm{ml} \mathrm{IL-23}$. Supernatants were collected after 96 hours and assayed for IL-4, IFN- $\gamma$ (Ab pairs from BD Biosciences - Pharmingen), and IL-17 by ELISA kit (R\&D Systems) according to the manufacture's directions. For IFN- $\gamma$ assay, $\mathrm{CD}^{+}$splenocytes were purified using CD4-specific magnetic beads on an automated magnetic cell sorter (Miltenyi Biotec) following the manufacturer's instructions. Cells were cultured for 72 hours with $1 \mu \mathrm{g} / \mathrm{ml}$ anti-CD28 (BD Biosciences - Pharmingen) on anti-CD3coated plates. IFN- $\gamma$ was assessed in supernatants by fluorescent bead array using IFN- $\gamma$-specific beads (Upstate USA Inc.) on a Luminex 100 analyzer instrument (Luminex Corp.) according to the manufacturer's recommendation. For proliferation assays, $\mathrm{T}$ cells were cultured on antiCD3-coated plates supplemented with IL-2 or with IL-23 and neutral- izing anti-IL-2 mAb. Cell cultures were pulsed after 96 hours with $1 \mu \mathrm{Ci}$ $\left[{ }^{3} \mathrm{H}\right]$ thymidine per well for 24 hours. Incorporation was measured by liquid scintillation spectroscopy, and data are expressed as mean counts per minute of triplicate wells. DCs were obtained from spleens of Rag-KO mice by depleting macrophages (F4/80) and erythrocytes by magnetic bead depletion as described above. The remaining cells were stained with anti-I-A, anti-CDllc, and anti-Mac-1 mAbs and sorted to greater than $95 \%$ purity. Cells were cultured with LPS $(10 \mu \mathrm{g} / \mathrm{ml})$ and/or IL-23 $(50 \mathrm{ng} / \mathrm{ml})$. Cell supernatants were harvested after 72 hours and assayed by ELISA. For intracellular cytokine analysis, memory $\mathrm{CD} 4^{+} \mathrm{T}$ cells were stimulated with PMA and ionomycin for 4 hours. Brefeldin A (EPICENTRE Biotechnologies) was adding during the last 2 hours of stimulation. Cells were harvested, fixed in $4 \%$ formaldehyde, permeabilized with $0.1 \%$ saponin, and then stained with FITC- or PE-conjugated anti-cytokine or isotype control mAb (BD Biosciences - Pharmingen). Cells were analyzed using a FACSCalibur and CellQuest software (BD).

RNA expression analysis. Total RNA was prepared from frozen colon tissue samples or from activated $\mathrm{CD} 4{ }^{+} \mathrm{CD} 45 \mathrm{RB}^{\text {low }} \mathrm{T}$ cells using RNeasy kit (QIAGEN) according to the manufacturer's protocols. Total RNA $(5 \mu \mathrm{g})$ was treated with DNAse I (Ambion Inc.) to remove contaminating genomic DNA, then reverse transcribed with Superscript II reverse transcriptase (Invitrogen Corp.) with oligo p(DT) ${ }_{15}$ (Roche Applied Science) and random hexamers (Promega), according to the manufacturer's protocol. Gene expression levels were determined by real-time quantitative PCR using an ABI 5700 sequence detector system (Applied Biosystems) and SYBR Green PCR Master Mix according to the manufacturer's instructions (Applied Biosystems). Primers were designed to ensure no cross-reactivity with other genes and, where possible, were designed to span intron/exon boundaries. PCR amplification of the housekeeping gene ubiquitin was performed for each sample to control for loading and to allow normalization between samples. Both water and genomic DNA controls were included to ensure specificity. Each data point was evaluated for integrity by analysis of the amplification plot and dissociation curves. Data normalized to ubiquitin were then compared and expressed as the fold induction of gene expression in treated samples versus control samples.

In vivo administration of $m A$ bs. Rag-KO recipient mice were injected i.p. with $2 \mathrm{mg}$ /mouse of isotype control (rat IgG1 GL113), anti-IL-17 (rat anti-mIL-17 IgG1 18H10), anti-IL-6 (rat anti-mIL-6 IgG1 MP5-20F3), or anti-IL-6 and anti-IL-17 combination mAb 1 day prior to naive IL-10-KO $\mathrm{T}$ cell reconstitution. Following $\mathrm{T}$ cell reconstitution, mice were dosed i.p. with $1 \mu \mathrm{g} /$ mouse IL-23 protein daily and $2 \mathrm{mg} /$ mouse total Ab weekly for 6 weeks. The health of mice was monitored daily to observe any gross signs of colitis (wasting and diarrhea).

Statistics. Unpaired Student's $t$ test was used to analyze data. Differences were considered significant at $P<0.05$.

\section{Acknowledgments}

We gratefully acknowledge Jim Cupp and the Schering-Plough Biopharma FACS Facility. We also thank C. Hannum, G. Zurawski, and J. Sedgwick for their helpful comments. Schering-Plough Biopharma is supported by Schering-Plough Corp.

Received for publication February 24, 2004, and accepted in revised form February 7, 2006.

Address correspondence to: Robert A. Kastelein, Schering-Plough Biopharma, 901 California Avenue, Palo Alto, California 94304, USA; Phone: (650) 496-1271; Fax: (650) 496-1200; E-mail: rob. kastelein@dnax.org. 
David Yen, Jeanne Cheung, and Heleen Scheerens contributed equally to this work.

David Yen's present address is: Roche Palo Alto, Palo Alto, California, USA.

1. Braegger, C.P. 1994. Immunopathogenesis of chronic inflammatory bowel disease. Acta Paediatr. Suppl. 83:18-21.

2. Berg, D.J., et al. 1996. Enterocolitis and colon cancer in interleukin-10-deficient mice are associated with aberrant cytokine production and CD4(+) TH1-like responses. J. Clin. Invest. 98:1010-1020.

3. Davidson, N.J., et al. 1996. Thelper cell 1-type CD4+ $\mathrm{T}$ cells, but not B cells, mediate colitis in interleukin 10-deficient mice. J. Exp. Med. 184:241-251.

4. Davidson, N.J., etal. 1998. IL-12, but not IFN-gamma, plays a major role in sustaining the chronic phase of colitis in IL-10-deficient mice. J. Immunol. 161:3143-3149.

5. Neurath, M.F., et al. 1995. Antibodies to interleukin 12 abrogate established experimental colitis in mice. J. Exp. Med. 182:1281-1290.

6. Cua, D.J., et al. 2003. Interleukin-23 rather than interleukin-12 is the critical cytokine for autoimmune inflammation of the brain. Nature. 421:744-748.

7. Murphy, C.A., et al. 2003. Divergent pro- and antiinflammatory roles for IL-23 and IL-12 in joint autoimmune inflammation. J. Exp. Med. 198:1951-1957.

8. Langrish, C.L., et al. 2005. IL-23 drives a pathogenic $\mathrm{T}$ cell population that induces autoimmune inflammation. J. Exp. Med. 201:233-240.

9. Mannon, P.J., et al. 2004. Anti-interleukin-12 antibody for active Crohn's disease. N. Engl. J. Med. 351:2069-2079.

10. Kauffman, C.L., et al. 2004. A phase I study evaluating the safety, pharmacokinetics, and clinical response of a human IL-12 p40 antibody in subjects with plaque psoriasis. J. Invest. Dermatol. 123:1037-1044.

11. Powrie, F., et al. 1994. Inhibition of Th1 responses prevents inflammatory bowel disease in scid mice reconstituted with CD45RBhi CD4+ T cells. Immunity. 1:553-562.

12. Yao, Z., et al. 1995. Human IL-17: a novel cytokine derived from T cells. J. Immunol. 155:5483-5486.

13. Shin, H.C., et al. 1999. Expression of IL-17 in human memory CD45RO+ T lymphocytes and its regulation by protein kinase A pathway. Cytokine. 11:257-266.

14. Fossiez, F., et al. 1998. Interleukin-17. Int. Rev. Immunol. 16:541-551.

15. Parham, C., et al. 2002. A receptor for the heterodimeric cytokine IL-23 is composed of IL-12Rbeta1 and a novel cytokine receptor subunit, IL-23R. Immunol. 16:365-396.

Jeanne Cheung and Heleen Scheerens's present address is: Genentech Inc., South San Francisco, California, USA.

The research was carried out at DNAX, which is now ScheringPlough Biopharma.

J. Immunol. 168:5699-5708.

16. Yamamoto, M., et al. 2000. IL-6 is required for the development of Th1 cell-mediated murine colitis. J. Immunol. 164:4878-4882.

17. Atreya, R., et al. 2000. Blockade of interleukin 6 trans signaling suppresses T-cell resistance against apoptosis in chronic intestinal inflammation: evidence in Crohn disease and experimental colitis in vivo. Nat. Med. 6:583-588.

18. Ito, H., et al. 2002. Anti-IL-6 receptor monoclonal antibody inhibits leukocyte recruitment and promotes T-cell apoptosis in a murine model of Crohn's disease. J. Gastroenterol. 37(Suppl. 14):56-61.

19. Gross, V., et al. 1992. Evidence for continuous stimulation of interleukin- 6 production in Crohn's disease. Gastroenterology. 102:514-519.

20. Fujino, S., et al. 2003. Increased expression of interleukin 17 in inflammatory bowel disease. Gut. 52:65-70.

21. Hata, K., et al. 2002. IL-17 stimulates inflammatory responses via NF-kappaB and MAP kinase pathways in human colonic myofibroblasts. Am. J. Physiol. Gastrointest. Liver Physiol. 282:G1035-G1044.

22. Oppmann, B., et al. 2000. Novel p19 protein engages IL-12p40 to form a cytokine, IL-23, with biological activities similar as well as distinct from IL-12. Immunity. 13:715-725.

23. Trinchieri, G. 1998. Proinflammatory and immunoregulatory functions of interleukin-12. Int. Rev.

24. Reimann, J., et al. 1993. Selective engraftment of memory CD4+ T cells with an unusual recirculation pattern and a diverse $T$ cell receptor- $V$ beta repertoire into scid mice. Eur. J. Immunol. 23:350-356.

25. Becker, C., et al. 2003. Constitutive p40 promoter activation and IL-23 production in the terminal ileum mediated by dendritic cells. J. Clin. Invest. 112:693-706. doi:10.1172/JCI200317464.

26. Witowski, J., et al. 2000. IL-17 stimulates intraperitoneal neutrophil infiltration through the release of GRO alpha chemokine from mesothelial cells. J. Immunol. 165:5814-5821.

27. Linden, A., Hoshino, H., and Laan, M. 2000. Airway neutrophils and interleukin-17. Eur. Respir. J. 15:973-977.

28. Laan, M., et al. 1999. Neutrophil recruitment by human IL-17 via C-X-C chemokine release in the airways. J. Immunol. 162:2347-2352.

29. Chabaud, M., et al. 1999. Human interleukin-17: a
T cell-derived proinflammatory cytokine produced by the rheumatoid synovium. Arthritis Rheum. 42:963-970.

30. Kotake, S., et al. 1999. IL-17 in synovial fluids from patients with rheumatoid arthritis is a potent stimulator of osteoclastogenesis. J. Clin. Invest. 103:1345-1352.

31. Infante-Duarte, C., et al. 2000. Microbial lipopeptides induce the production of IL-17 in Th cells. J. Immunol. 165:6107-6115.

32. Malfait, A.M., et al. 1998. Blockade of IL-12 during the induction of collagen-induced arthritis (CIA) markedly attenuates the severity of the arthritis. Clin. Exp. Immunol. 111:377-383.

33. Matthys, P., et al. 1998. Anti-IL-12 antibody prevents the development and progression of collagen-induced arthritis in IFN-gamma receptor-deficient mice. Eur. J. Immunol. 28:2143-2151.

34. Joosten, L.A., et al. 1997. Dual role of IL-12 in early and late stages of murine collagen type II arthritis. J. Immunol. 159:4094-4102.

35. Lubberts, E., et al. 2001. IL-1-independent role of IL-17 in synovial inflammation and joint destruction during collagen-induced arthritis. J. Immunol. 167:1004-1013.

36. Lenarczyk, A., et al. 2000. Antigen-induced IL-17 response in the peripheral blood mononuclear cells (PBMC) of healthy controls. Clin. Exp. Immunol. 122:41-48.

37. Aggarwal, S., et al. 2003. Interleukin-23 promotes a distinct $\mathrm{CD} 4 \mathrm{~T}$ cell activation state characterized by the production of interleukin-17. J. Biol. Chem. 278:1910-1914.

38. Pasare, C., and Medzhitov, R. 2003. Toll pathway-dependent blockade of CD4+CD25+ T cellmediated suppression by dendritic cells. Science. 299:1033-1036.

39. Belladonna, M.L., et al. 2002. IL-23 and IL-12 have overlapping, but distinct, effects on murine dendritic cells. J. Immunol. 168:5448-5454.

40. Simpson, S.J., et al. 1998. T cell-mediated pathology in two models of experimental colitis depends predominantly on the interleukin 12 /Signal transducer and activator of transcription (Stat)-4 pathway, but is not conditional on interferon gamma expression by T cells. J. Exp. Med. 187:1225-1234.

41. Braegger, C.P. 1994. Immunopathogenesis of chronic inflammatory bowel disease. Acta Paediatr. Suppl. 83:18-21. 